\title{
Early-phase vascular involvement is associated with acute pancreatitis severity: a magnetic resonance imaging study
}

\author{
Zhi-Qiong Jiang ${ }^{1}$, Bo Xiao ${ }^{2,3}$, Xiao-Ming Zhang ${ }^{2}$, Hai-Bo Xu ${ }^{3}$ \\ ${ }^{1}$ Department of Geratology, Affiliated Hospital of North Sichuan Medical College, Nanchong, China; ${ }^{2}$ Department of Radiology, Affiliated Hospital \\ of North Sichuan Medical College, North Sichuan Medical College, Nanchong, China; ${ }^{3}$ Department of Radiology, Zhongnan Hospital of Wuhan \\ University, Wuhan University, Wuhan, China
}

Correspondence to: Xiao Bo, MD, PhD. Department of Radiology, Affiliated Hospital of North Sichuan Medical College, Nanchong 637000, China; Department of Radiology, Zhongnan Hospital of Wuhan University, Wuhan University, Wuhan 430071, China. Email: xiaoboimaging@163.com.

Background: Although a number of studies have reported on the vascular abnormalities detected by magnetic resonance imaging (MRI) in patients with late-phase acute pancreatitis (AP), few have studied those occurring in the early phase of the disease. The aim of this research was to investigate the MRI findings of early vascular abnormalities in AP and to analyze the correlation of the prevalence of vascular involvement with the severity of AP based on the MR severity index (MRSI) and Acute Physiology and Chronic Health Evaluation (APACHE) II scores.

Methods: A retrospective analysis was conducted of 301 consecutive AP patients who were admitted to our institution between March 2013 and June 2019. All patients underwent initial MRI during the early phase of pancreatitis and one or more repeat MRI scans in the late phase. Peripancreatic vascular conditions and pancreatitis were assessed using T1-/T2-weighted imaging and dynamic-enhanced MRI. The association between the prevalence of vascular involvement and AP severity graded according to the MRSI or APACHE II score was analyzed using Spearman's rank correlation.

Results: Among 301 AP patients, 75 (24.9\%) had at least one MRI-detected vascular abnormality. Overall, vascular involvement on MRI was higher in necrotizing pancreatitis than in edematous pancreatitis [43.2\% (54/125) vs. $\left.11.9 \%(21 / 176), \chi^{2}=38.2, \mathrm{P}<0.001\right]$. In the early phase of AP, the prevalence of splenic vein phlebitis, portal vein phlebitis, and splenic arterial arteritis was $24.9 \%$ (75/301), $22.3 \%$ (67/301), and $19.9 \%$ (60/301), respectively. Splenic vein phlebitis was seen on initial MRI in $55.6 \%(15 / 27)$ of patients who had splenic vein thrombosis on repeat MRI. The MRSI scores showed that the prevalence of splenic vein phlebitis, portal vein phlebitis, and splenic arterial arteritis, respectively, was correlated with the severity of pancreatitis $(\mathrm{r}=0.532,0.487$, and 0.456 ; all $\mathrm{P}<0.01)$. The APACHE II scores showed that the prevalence of MRI-detected vascular involvement was significantly correlated with AP severity $(r=0.335, \mathrm{P}<0.05)$.

Conclusions: Vascular abnormalities, including splenic vein phlebitis and splenic arterial arteritis, are commonly seen on MRI in patients with early-phase AP, and they may be supplementary indicators that can reflect the severity of pancreatitis.

Keywords: Acute pancreatitis; vascular complication; splenic vein phlebitis; splenic arterial arteritis; magnetic resonance imaging (MRI)

Submitted Feb 13, 2020. Accepted for publication Nov 24, 2020.

doi: 10.21037/qims-20-280

View this article at: http://dx.doi.org/10.21037/qims-20-280 


\section{Introduction}

Acute pancreatitis (AP) is the most common gastrointestinal disease, which can cause various systemic and local complications during and after an episode (1-4). The clinical course of moderately severe or severe disease usually comprises two phases: an early phase lasting approximately 1 week and a late phase commencing 1 week after onset (1-3). The 2012 revised Atlanta classification describes four local complications of pancreatic/peripancreatic fluid collection during AP including acute peripancreatic fluid collection, acute necrotic collection, pancreatic pseudocyst, and walledoff necrosis $(1,5)$. However, additional local complications of AP, such as peripancreatic vascular involvement, are not reported in the classification. In our experience, early-phase vascular abnormalities in $\mathrm{AP}$ are not rare in clinical practice. Vascular involvement in pancreatitis can be dangerous and potentially lethal; thus, its early recognition and diagnosis are of great importance to radiologists (6-10).

Compared to computerized tomography (CT), highfield-strength magnetic resonance imaging (MRI) is a safe and effective noninvasive imaging modality to diagnose and evaluate $\mathrm{AP}$ without radiation hazard and the risk of iodinated allergic reactions (8-10). With its superior tissue resolution, MRI is now considered to be the front-line imaging method for the assessment of pancreatitis and its local complications, including the detection of hemorrhage (8).

To the best of our knowledge, previous studies $(5-7,11)$ have mainly reported on late-stage vascular conditions during AP using CT. In contrast, data regarding vascular abnormalities on MRI in early-phase AP patients are lacking, and the relationships between vascular abnormalities and disease severity in these patients have remained unclear. Therefore, this study aimed to determine the prevalence of early-phase vascular involvement in AP using MRI, and to investigate its correlation with the severity of AP based on the MR severity index (MRSI) and Acute Physiology and Chronic Health Evaluation (APACHE) II system.

\section{Methods}

\section{Patient selection}

This study was conducted in accordance with the Declaration of Helsinki (as revised in 2013) and was approved by the institutional ethics board of the Affiliated Hospital of North Sichuan Medical College [No. 2017ER(A)026]. Patient informed consent was waived due to the retrospective design of the study.

The study population comprised 301 consecutive adult patients (aged $\geq 18$ years) with AP who were admitted to our hospital between March 2013 and June 2019. The diagnostic criteria for AP were based on the 2012 revised Atlanta classification (1). The inclusion criteria were: (I) in-patients with AP who had complete clinical data and laboratory test results including amylase and lipase; (II) underwent an in-patient initial MRI scan during the early phase of pancreatitis ( $<7$ days after symptom onset) and had one or more repeat MRI scans during the late phase ( $\geq 7$ days after symptom onset) for clinical evaluation of local complications and to determine prognosis. Our hospital is a specialized institution for pancreatic diseases, and MRI is performed for all in-patients with AP (both interstitial edema pancreatitis and necrotizing pancreatitis) providing there are no contraindications to its use. The exclusion criteria included: (I) admission $\geq 7$ days after symptom onset $(\mathrm{n}=21)$; (II) recurrent pancreatitis, chronic pancreatitis, or pancreatic cancer $(\mathrm{n}=33)$; (III) pregnancy $(\mathrm{n}=5)$; (IV) cirrhosis or other systemic tumors or coagulopathy disorders $(\mathrm{n}=13)$; (V) heavy motion artifacts of images $(\mathrm{n}=12)$; and (VI) a history of pseudoaneurysm $(\mathrm{n}=0)$. There were no cases of post-Endoscopic Retrograde Cholangiopancreatography (ERCP) pancreatitis or renal neoplastic disorder among the AP patients in this study.

\section{Data collection and MRI protocols}

The following data were collected: (I) general characteristics, including sex, age, etiologies of AP, APACHE II score during the 48 hours following admission, and MRSI score based on the initial MRI scan. The common causes of AP include solid gallstones in the gallbladder and common bile duct, a history of alcohol abuse before AP onset, and hyperlipidemia (increased fasting triglyceride levels $>1,000 \mathrm{mg} / \mathrm{dL}$ ) (1-5). (II) MRI-detected vascular abnormalities in AP as indicated below.

All MRI examinations were performed using a 3.0T MR scanner (GE Signa Discovery MR 750, USA). MRI sequences and scanning parameters included: (I) 3-dimensional (3D) liver acquisition with volume acceleration-flexible (3D-LAVA-flex) T1-weighted imaging with fat suppression: repetition time (TR)/echo time (TE) $=$ (3.6-4.4) $\mathrm{ms} /(1.7-1.9) \mathrm{ms}$, section thickness/intersection gap $=5 \mathrm{~mm} / 0 \mathrm{~mm}$, matrix $=224 \times 192$, field of view $(\mathrm{FOV})=36 \times$ $36 \mathrm{~cm}$, flip angle $=12^{\circ}$, and number of excitations $=0.75$; (II) axial fast-recovery fast spin-echo (FRFSE) T2-weighted 
imaging with fat suppression: TR/TE $=(2,500-3,000) \mathrm{ms} /$ $(90-110) \mathrm{ms}$, section thickness/intersection gap $=6 \mathrm{~mm} / 1 \mathrm{~mm}$, matrix $=384 \times 384, \mathrm{FOV}=34 \mathrm{~cm} \times 34 \mathrm{~cm}$; (III) axial and coronal single-shot fast spin-echo (SSFSE) T2-weighted imaging: TR/TE $=(4,500-6,000) \mathrm{ms} /(90-120) \mathrm{ms}$, section thickness/intersection gap $=5 \mathrm{~mm} / 1 \mathrm{~mm}$, matrix $=384 \times 256$, and FOV $=36 \times 36 \mathrm{~cm}$; (IV) SSFSE radial series slab MR cholangiopancreatography (MRCP): TR/TE $=(4,000-$ $5,000) \mathrm{ms} /(900-1,000) \mathrm{ms}$, section thickness $=50 \mathrm{~mm}$, matrix $=384 \times 256, \mathrm{FOV}=34 \mathrm{~cm} \times 34 \mathrm{~cm}$; (V) 3D-LAVAflex dynamic contrast-enhancement with fat suppression: $0.2 \mathrm{mmol} / \mathrm{L}$ per kilogram of gadolinium (Schering Guangzhou Co., China) was intravenously injected at a rate of $3.0 \mathrm{~mL} / \mathrm{s}$ with a high-pressure injector (Spectris MR Injection System, Medrad Inc., USA), followed by a $20 \mathrm{~mL}$ saline solution flush. Arterial, venous, and delayed phase scans were then obtained $25-30,55-60 \mathrm{~s}$ after the injection, and $120 \mathrm{~s}$ after the injection, respectively.

\section{Definitions and image review}

$\mathrm{AP}$ was classified as interstitial edematous or necrotizing pancreatitis based on the MRI features (10). Pancreatic necrosis was defined as focal or diffuse non-enhancement of the parenchymal gland of the pancreas $(11,12)$. When present, the degree of parenchymal necrosis $(<30 \%$, $30-50 \%$, or $>50 \%$ ) was recorded. Extrapancreatic necrosis was determined by extrapancreatic changes exceeding fat stranding in the absence of findings of non-enhanced pancreatic parenchyma on contrast-enhanced images (12). Based on published articles $(13,14)$, the severity of AP was categorized as mild (0-3 points), moderate (4-6 points), or severe (7-10 points) using the MRSI score. APACHE II scores were used to grade the severity of AP as mild or severe, with the latter defined by a score $\geq 8$ (1). The 2012 revised Atlanta classification was not adopted as the standard criterion for AP severity, because as a new standard, it is not yet used routinely in clinical practice.

To evaluate vascular involvement on MRI, the detected imaging characteristics of peripancreatic major vasculature, both arteries and veins, were visualized. A wide range of vascular pathologies were reported, including the following: (I) venous involvement/phlebitis, which was defined as linear or patchy hyperintensity within peripancreatic veins on T2weighted imaging with fat suppression, with the involved vein segments showing blurred and irregular edges (10). In addition, as the normal diameter of the splenic vein ranges from 6 to $8 \mathrm{~mm}$, values $<6 \mathrm{~mm}$ were considered as splenic vein stenosis $(15,16)$. (II) Arterial involvement/ arteritis, which was defined as focal hyperintensity within peripancreatic arteries on $\mathrm{T} 2$-weighted imaging with fat suppression, with the involved arterial segments showing unsharp and blurred edges, as well as poor enhancement on contrast-enhanced arterial phase images $(8,10)$. In addition, hemorrhage was defined as patchy hyperintensity in peripancreatic areas on T1-weighted imaging with fat suppression $(8,10)$. (III) Venous thrombosis, which was diagnosed based on an intravenous filling defect within the lumens of peripancreatic veins in the contrast-enhanced venous phase. (IV) Portal hypertension/varices, which was defined as portal vein enlargement (a diameter $>1.3 \mathrm{~cm}$ ) and increased collateral vessels. Variceal channels of venous drainage included gastric, gastrosplenic, and omental varices $(16,17)$. (V) Pseudoaneurysm, which was defined as a cavity communicated within the peripancreatic artery, with the enhancement of the cavity similar to the abdominal aorta on the arterial phase of dynamic-enhanced images $(9,10)$. Theoretically, the gold standard for evaluating earlyand late-phase vascular involvement is digital subtraction angiography (DSA). However, DSA is invasive for AP patients, and it is not realistic to perform this test in patients with severe pancreatitis. Therefore, we identified early- and late-phase vascular involvement related to pancreatitis based on MRI findings.

All MRI images were independently assessed by two radiologists ( $\mathrm{A}$ and $\mathrm{B}$, with $>12$ and $>20$ years of experience in abdominal MRI, respectively). Both radiologists were aware that their role was to evaluate severity and vascular abnormality, and that the patients had AP. In cases of discrepancy, a consensus was reached through discussion. Following this, data analysis and measurement were performed by A based on the consensus findings.

\section{Statistical analysis}

The Kolmogorov-Smirnov test was used for data normality. Normally distributed continuous variables were presented as mean $\pm \mathrm{SD}$, and data with skewed distribution were expressed as the median. Categorical variables were presented as percentages and compared using the $\chi^{2}$ test or Fisher's exact test. Interobserver agreement for the evaluation of peripancreatic vascular abnormalities was calculated using the kappa statistic. Furthermore, the correlation coefficient of the prevalence of peripancreatic vascular involvement with the severity of pancreatitis based on the MRSI or APACHE II score was calculated using 
Table 1 Demographic information of AP patients ( $\mathrm{N}=301)$

\begin{tabular}{lc}
\hline Variables & Values \\
\hline Gender (men/women): & $148 / 153$ \\
Age (years), mean [SD], range & $52[12], 18-67$ \\
Etiology, n (\%) & \\
Gallstone-related & $170(56.5)$ \\
Hyperlipidemia-related & $53(17.6)$ \\
Alcohol-related & $51(16.9)$ \\
Surgery-related & $12(4.0)$ \\
Cholangio-pancreatic duct variants & $7(2.3)$ \\
Unknown & $8(2.7)$ \\
MRSI score, mean \pm SD [range] & $3.8 \pm 1.7[1-10]$ \\
APACHE II score, mean \pm SD [range] & $7.0 \pm 4.8[0-19]$ \\
\hline AP, acute pancreatitis; MRSI, MR severity index; APACHE, Acute \\
Physiology and Chronic Health Evaluation.
\end{tabular}

Spearman's rank correlation $(5,8,16)$. Data were analyzed using SPSS statistical software (v16.0, SPSS Inc., Chicago, USA), and a $P$ value $<0.05$ was considered to show a significant difference.

\section{Results}

Baseline characteristics of the 301 AP patients including sex, age, etiology, MRSI score, and APACHE II score are shown in Table 1. Transient organ failure was seen in $62(20.6 \%)$ patients, and $23(7.6 \%)$ patients experienced persistent organ failure. The mean length of hospital stay was $18.9 \pm 10.6$ days (8-94 days).

\section{AP patterns on MRI and its severity based on MRSI}

Among the 301 patients with AP, MRI was repeated once in 246 patients and twice in 55 patients. Based on the initial MRI images after hospitalization, 176 patients $(58.5 \%)$ had edematous pancreatitis, and 125 (41.5\%) had necrotizing pancreatitis (including 120 patients with pancreatic necrosis combined with extrapancreatic necrosis and 5 patients with extrapancreatic necrosis only). Of the 120 cases of parenchymal necrosis, $<30 \%, 30-50 \%$, and $>50 \%$ parenchymal necrosis was detected in $87(72.5 \%)$, $18(15.0 \%)$, and $15(12.5 \%)$ patients, respectively. The mean MRSI score was 3.8 points (SD, 1.7; range, 1-10) for the $301 \mathrm{AP}$ patients, 2.7 (SD, 0.8) for the edematous pancreatitis patients, and $5.3(\mathrm{SD}, 1.7)$ for those with necrotizing pancreatitis. According to the MRSI score, mild, moderate, and severe pancreatitis was seen in $46.8 \%$ (141/301), 44.6\% (134/301), and 8.6\% (26/301) of patients, respectively.

\section{MRI-detected vascular abnormalities in AP}

Overall, based on either initial or repeat MRI scans, $24.9 \%(75 / 301)$ of patients had at least one AP-associated peripancreatic vascular abnormality detected by MRI. There was good interobserver agreement between the two radiologists in the assessment of peripancreatic vascular involvement (all Kappa $>0.850$ ). The prevalence of peripancreatic vascular involvement on MRI was higher among necrotizing pancreatitis than among edematous pancreatitis [43.2\% (54/125) vs. $11.9 \%(21 / 176), \chi^{2}=38.2$, $\mathrm{P}<0.001]$.

\section{Early-phase vascular abnormalities on initial MRI}

The specific details of the detection time and rate of initial MRI are listed in Table 2. Splenic vein involvement/phlebitis was the most common finding on $\mathrm{T} 2$-weighted images with fat suppression (Figure 1) and manifested as patchy or linear hyperintensity within an involved vein segment (loss of normal vessel flowing void effect), an irregular venous endothelium plane with blurred vein edges, and linear hyperintensity located along the involved vein. Splenic, portal, and mesenteric superior vein phlebitis was seen in $24.9 \%$ (75/301), $22.3 \%$ (67/301), and $15.3 \%$ (46/301) of patients, respectively. Of the 75 patients with splenic vein phlebitis, $44.0 \%$ (33/75) had splenic vein stenosis (Figure 1) with a mean diameter of $3.5 \mathrm{~mm}$ (range, $2-5.5 \mathrm{~mm}$ ).

Splenic arterial involvement/arteritis (Figure 2), which manifests as irregular and blurred edges in the involved arterial segments, with loss of the normal vessel flowing void effect, was revealed in $19.9 \%(60 / 301)$ of patients on initial MRI. Splenic arterial arteritis complicating hemorrhage was seen in $6.3 \%$ (19/301) of patients as patchy hyperintensity in peripancreatic areas on T1-weighted images with fat suppression, as well as contrast extravasation from the proximal splenic artery or a splenic artery branch (Figure 2) during the contrast-enhanced arterial phase.

In addition, splenic vein thrombosis (Figure 2), portal vein thrombosis, mesenteric superior vein thrombosis, and portal hypertension/varices (Figure 2) were observed in $4.0 \%(12 / 301), 3.0 \%$ (9/301), $2.3 \%$ (7/301), and $4.0 \%$ $(12 / 301)$ of patients, respectively, on initial MRI contrast- 
Table 2 Incidence, MRI detection time, and MRI detection rate on initial or repeat MRI of vascular abnormalities in acute pancreatitis

\begin{tabular}{|c|c|c|c|c|}
\hline Vascular abnormalities & Incidence, \% [n] & $\begin{array}{l}\text { Mean time of onset to first } \\
\text { detection, days [range] }\end{array}$ & $\begin{array}{l}\text { MRI detection rate on initial } \\
\qquad \text { MRI, \% [n] }\end{array}$ & $\begin{array}{l}\text { MRI detection rate on repeat } \\
\text { scans, } \%[\mathrm{n}]\end{array}$ \\
\hline $\begin{array}{l}\text { Splenic arterial involvement } \\
\text { (arteritis) }\end{array}$ & $19.9[60]$ & $4.5[1-6]$ & $100.0[60]$ & 38.3 [23] \\
\hline $\begin{array}{l}\text { Splenic vein involvement } \\
\text { (phlebitis) }\end{array}$ & $24.9[75]$ & $4.6[1-6]$ & $100.0[75]$ & 32 [24] \\
\hline $\begin{array}{l}\text { Mesenteric superior vein } \\
\text { involvement (phlebitis) }\end{array}$ & $15.3[46]$ & $4.7[1-6]$ & $100.0[46]$ & $28.3[13]$ \\
\hline Splenic vein thrombosis & $9.0[27]$ & $14.1[5-35]$ & $44.4[12]$ & $55.6[15]$ \\
\hline Portal vein thrombosis & $7.3[22]$ & $15.4[5-35]$ & $40.9[9]$ & $59.1[13]$ \\
\hline Pseudoaneurysm & $1.7[5]$ & 34.6 [27-52] & $0[0]$ & $100.0[5]$ \\
\hline
\end{tabular}

MRI, magnetic resonance imaging.
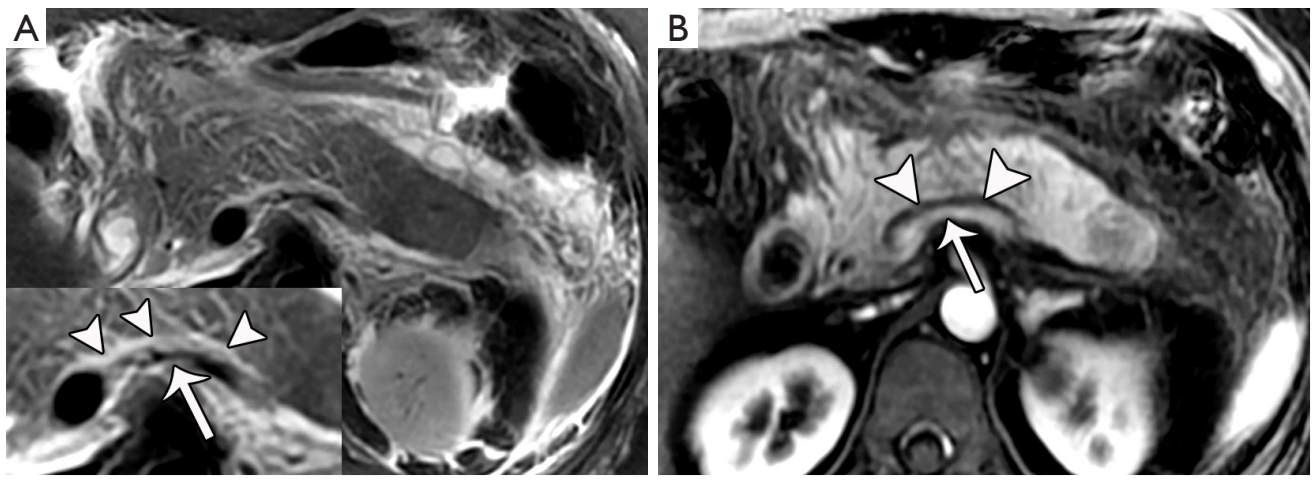

Figure 1 A 41-year-old woman with splenic vein phlebitis complicating splenic vein stenosis in the early phase of moderate acute pancreatitis. (A) MRI T2-weighted image with fat suppression performed 5 days after onset demonstrates patchy hyperintensity within the splenic vein (loss of normal flowing void effect) (arrow) and an irregular venous endothelium plane, as well as linear fluid hyperintensity (arrowheads) situated along the involved vein segment. (B) Note the normal enhancement of the splenic vein with stenosis (arrow) and a diameter of $3 \mathrm{~mm}$ at the contrast-enhanced venous phase. Non-enhancement of linear fluid signal (arrowheads) is also shown. MRI, magnetic resonance imaging.

enhanced images in the early phase of AP.

\section{Late-phase vascular conditions on repeat MRI}

The splenic vein phlebitis, portal vein phlebitis, and splenic arterial arteritis seen on initial MRI had disappeared on repeat MRI in $68.0 \%(51 / 75), 68.7 \%(46 / 67)$, and $61.7 \%$ (37/60) of patients, respectively.

Of the 27 patients with splenic vein thrombosis, $55.6 \%$
$(15 / 27)$ had splenic vein phlebitis on initial MRI imaging and newly complicated splenic vein thrombosis determined by repeat MRI (Figure 3), with a mean detection time of 20.9 (SD, 7.5) days (13-35 days). Of the patients with splenic vein thrombosis detected by initial MRI, 44.4\% $(12 / 27)$ had portosystemic thrombus absorption visualized on repeat MRI in the late phase of AP (Figure 4).

In addition, $1.7 \%(5 / 301)$ of patients who had splenic 

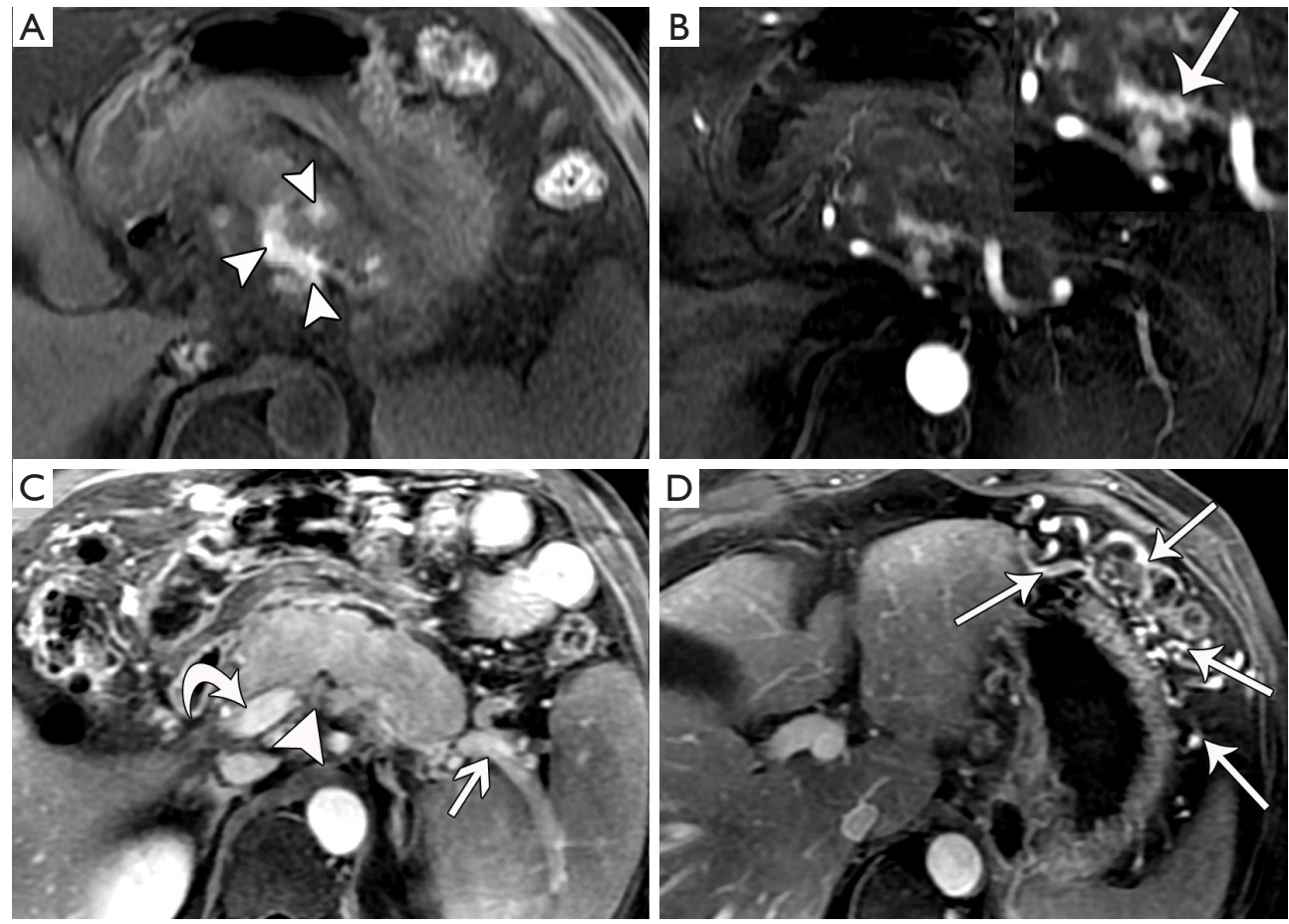

Figure 2 A 42-year-old male with splenic arterial involvement/arteritis complicating spontaneous hemorrhage, splenic vein thrombosis, and portal hypertension/varices in the early phase of severe acute pancreatitis. (A) MRI T1-weighted image with fat suppression performed 6 days after onset demonstrates patchy hyperintensity in peripancreatic areas (arrowheads). (B) Contrast-enhanced arterial phase image showing the irregular and blurred edge of the proximal splenic artery with contrast extravasation (arrow). (C) Venous phase image identifying intraluminal thrombus in the splenic vein (arrowhead). Dilation of the portal vein (1.4 cm in diameter, curved arrow) and distal splenic vein (1.2 cm in diameter, straight arrow) can be seen. (D) Another venous phase image showing multiple enhancing varices (arrows) through the short gastric vein and gastroepiploic vein. MRI, magnetic resonance imaging.

arterial or arterial branch pseudoaneurysm (Figure 5) identified by repeat MRI had splenic arterial arteritis complicating splenic arterial or arterial branch hemorrhage determined by initial MRI images in the early phase of AP.

\section{Correlation of the incidence of vascular abnormalities with the severity of AP based on the MRSI or APACHE II score}

Among the $301 \mathrm{AP}$ patients, the prevalence of each form of vascular involvement in mild, moderate, and severe disease was statistically significant (all $\mathrm{P}<0.01$ ) (Figure 6). The detailed correlations of the incidence of peripancreatic vascular involvement in AP with disease severity according to the MRSI are shown in Table 3. According to the APACHE II score, the incidence of mild and severe AP was $62.8 \%(189 / 301)$ and $37.2 \%$ (112/301), respectively. The prevalence of vascular involvement in patients with mild and severe AP was $13.8 \%$ (26/189) and 43.7\% (49/112), respectively $\left(\chi^{2}=33.82, \mathrm{P}<0.001\right)$, and the correlation of the incidence of vascular involvement with AP severity on the basis of APACHE II score was statistically significant $(\mathrm{r}=0.335, \mathrm{P}<0.05)$.

\section{Discussion}

In this study, $24.9 \%$ of 301 patients showed evidence of at least one peripancreatic vascular abnormality associated with AP during MRI assessment. The prevalence of peripancreatic vascular involvement on MRI was higher in necrotizing pancreatitis than in edematous pancreatitis. Splenic vein phlebitis and splenic arterial involvement/ arteritis were common MRI findings in the early phase of AP. Splenic arterial arteritis complicating hemorrhage in the early phase of AP was seen in $6.3 \%$ of patients, and patients with splenic vein thrombosis determined by repeat MRI were found to have splenic vein phlebitis on initial MRI. We also found that the prevalence of early vascular involvement 

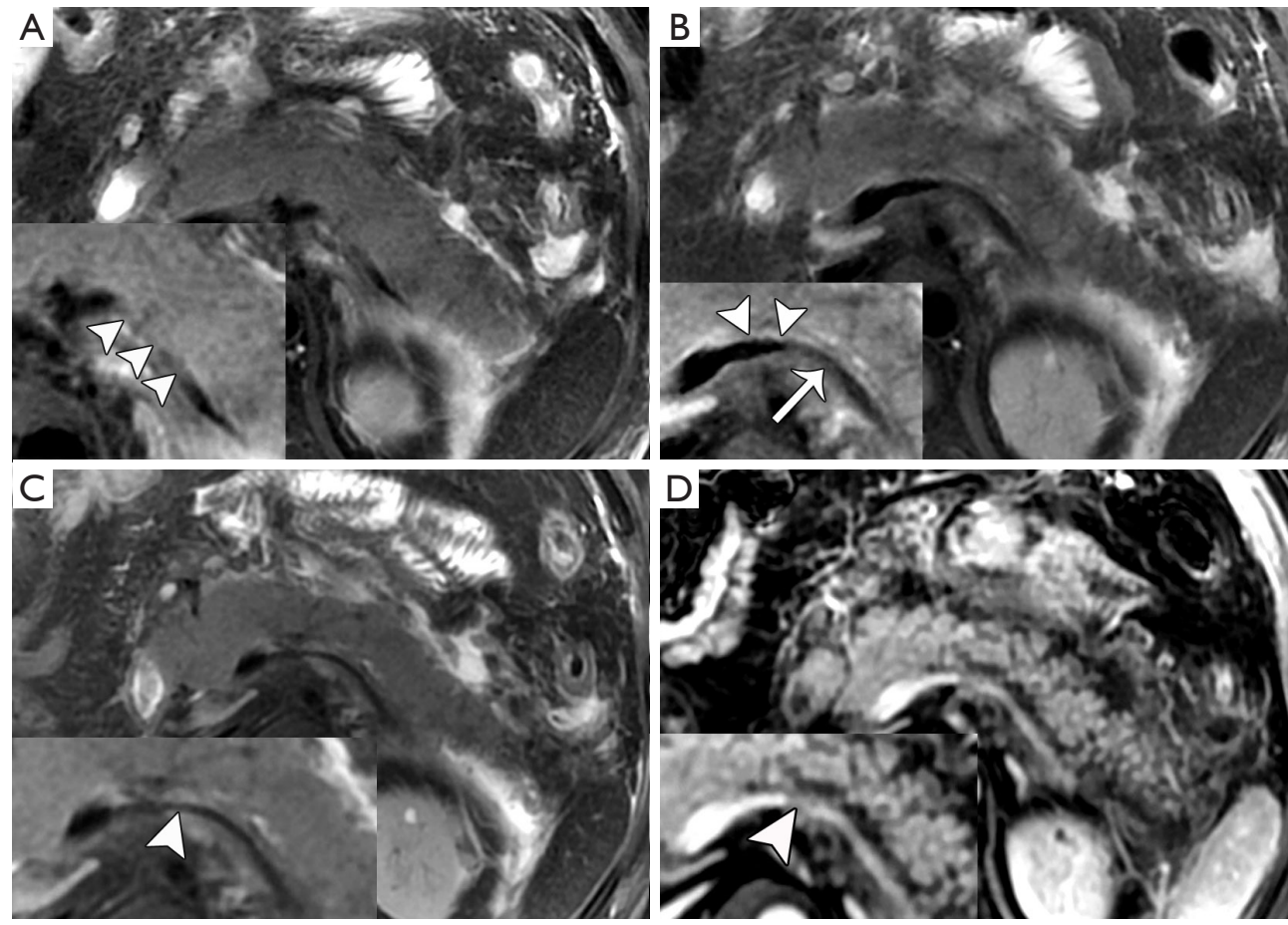

Figure 3 A 46-year-old male with splenic arteritis and venous phlebitis in the early phase of moderate acute pancreatitis and splenic venous thrombosis in the late phase of the disease. (A) MRI T2-weighted image with fat suppression performed 2 days after the onset of pancreatitis shows linear hyperintensity within the splenic artery (loss of normal flowing void effect) (arrowheads). Image (B) obtained at a lower level than (A). This image demonstrates an irregular endothelium line (arrowheads) of the involved splenic vein with intravenous hyperintensity (arrow). (C,D) MRI T2-weighted image (C) and contrast-enhanced venous phase image (D) performed 13 days after the onset of pancreatitis identifying splenic vein stenosis (arrowhead) with a diameter of $2 \mathrm{~mm}$, and an intravenous filling defect indicating the presence of thrombosis. MRI, magnetic resonance imaging.

in AP increased with the severity of pancreatitis based on MRSI or APACHE II score.

The main strength of this study is that we assessed earlyphase vascular conditions in AP patients by using MRI in the largest cohort to date. We used an initial MRI scan in the early phase of AP (performed $<7$ days of onset) and at least one repeat MRI in the late phase of AP. MRI proved to be superior in the detection of subtle, early-phase vascular abnormalities compared to CT, which has been used in prior studies. Previous studies have mainly focused on the CT detection of late-phase vascular complications, such as pseudoaneurysms $(7,11)$. In their study that used CT to evaluate patients within 1 week of AP onset, Mortelé et al. (7) reported that vein thrombosis and arterial hemorrhagic complications were found in $90 \%$ of cases. By using the superior tissue resolution of MRI, we found that vascular inflammatory changes were also relatively common in the early phase of pancreatitis, especially in peripancreatic veins.
Splenic vein phlebitis was seen most frequently (in $24.9 \%$ of patients), followed by portal vein phlebitis and mesenteric superior vein phlebitis. In addition, $44 \%$ of patients with splenic vein phlebitis had splenic vein stenosis in the early phase of AP. These new MRI findings may be explained by the close anatomic relationship between peripancreatic vasculatures and the pancreas itself, which results in local inflammatory spread and a weakened venous wall that is prone to collapse (17-20). Interestingly, following treatment, the MRI signs of portosplenomesenteric vein phlebitis disappeared in most of these patients, suggesting that portosystemic vein phlebitis might be considered as a complementary sign for treatment evaluation.

MRI indications of splenic arterial involvement/ arteritis were seen in $19.9 \%$ of patients in the early phase of pancreatitis. The appearance of an irregular and blurred edge of the involved arterial segment was likely due to direct corrosion of the arterial wall caused by the inflammatory 

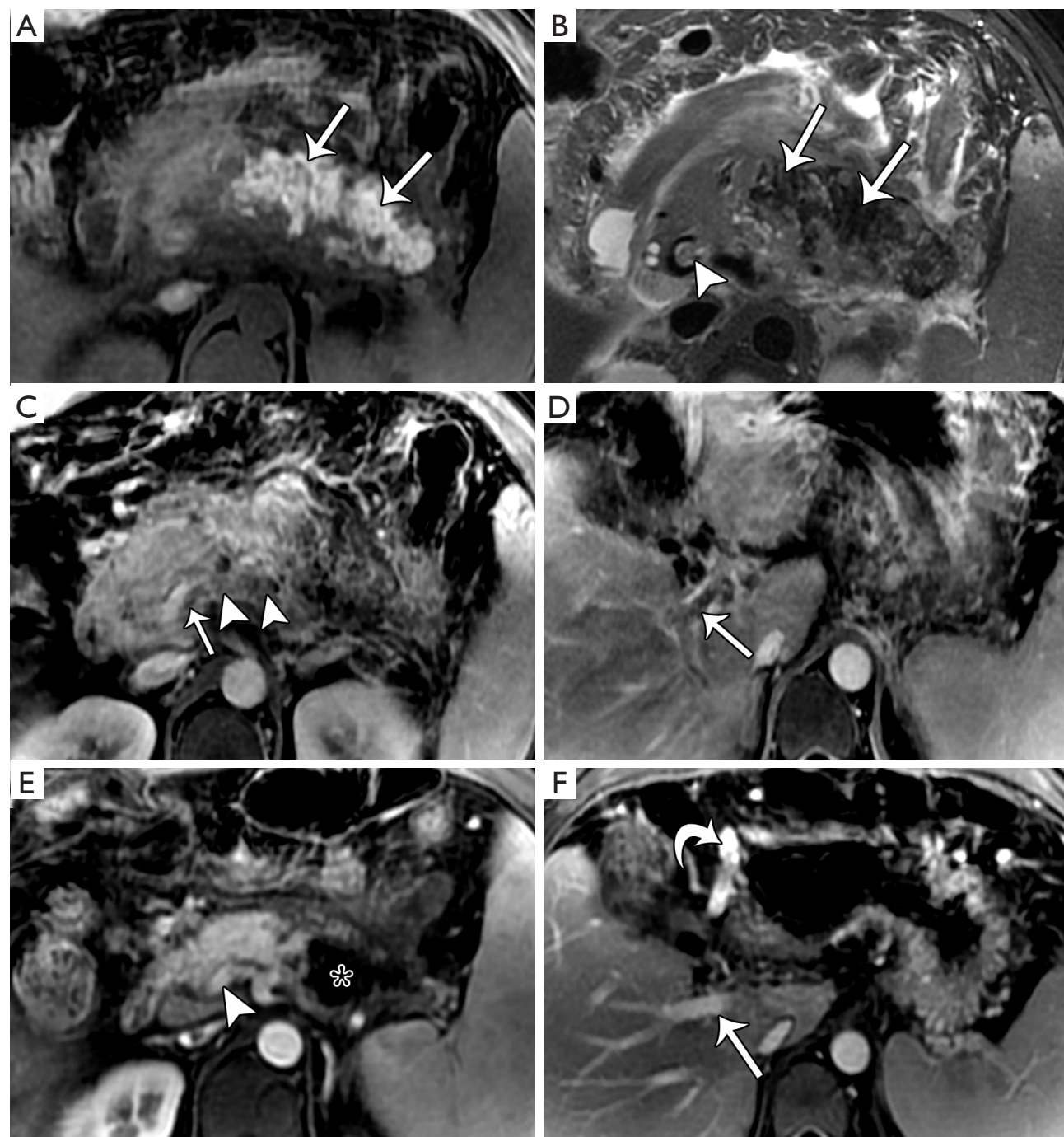

Figure 4 A 48-year-old man with vein thrombosis in the early phase of severe acute pancreatitis and thrombus absorption in the late stage. (A) MRI T1-weighted image with fat suppression performed 6 days after the onset of pancreatitis showing large areas of hyperintensity (arrows) occupying the body and tail of the pancreas. (B) T2-weighted image with fat suppression showing areas of hypointensity (arrows) and relative hyperintensity in the portal vein (arrowhead). (C,D) Contrast-enhanced venous phase images show the lack of opacification of the splenic vein (arrowheads) and the portal vein (arrows) consistent with vein thrombus. After treatment, post-contrast venous phase images (E,F) obtained 30 days after pancreatitis onset showing thrombus absorption of the splenic vein (arrowhead) and portal vein (arrow), as well as walled-off necrosis (asterisk) formation. Note a mildly enlarged right gastroepiploic vein (curved arrow) due to a history of splenic vein thrombosis. MRI, magnetic resonance imaging.

exudates of AP. Most importantly, we observed that $6.3 \%$ of our cohort had splenic arterial arteritis complicating splenic arterial or arterial branch hemorrhage in the early phase of AP. Also, patients with splenic arterial pseudoaneurysm identified by repeat MRI were found to have splenic arterial arteritis complicating hemorrhage on their initial MRI. These phenomena may be responsible for ongoing inflammation necrotizing to the splenic artery or its branch, and subsequent erosion or rupture of the arterial wall (21). This may explain why some AP patients experience clinical haemorrhage and we support the view that MRI can be used as an early diagnostic measure to assess the potential risk of hemorrhage in AP patients (8), after which treatment strategies, such as selective arterial angiography and 

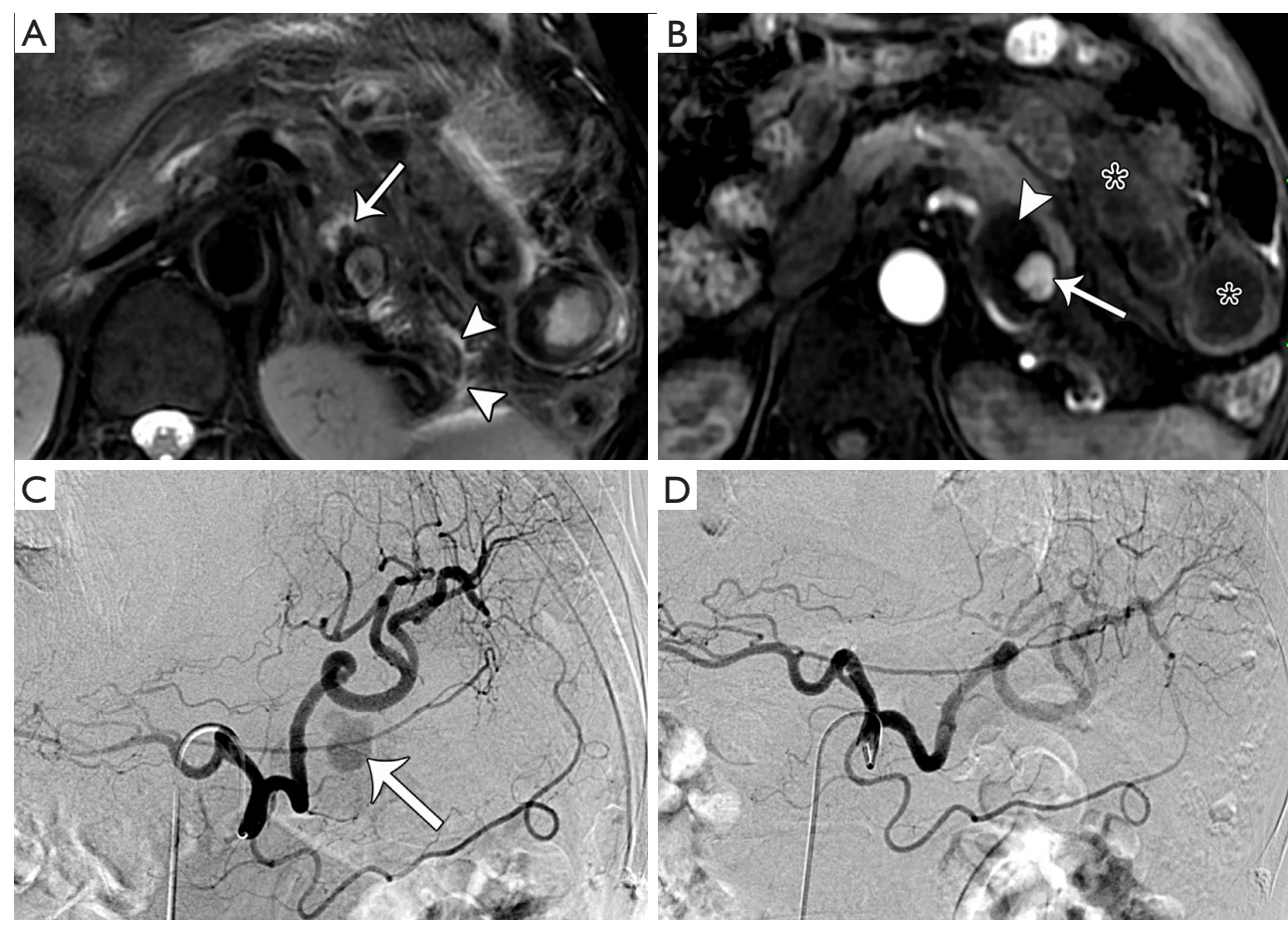

Figure 5 A 67-year-old male with a remote history of severe acute pancreatitis presented with splenic artery pseudoaneurysm before and after embolization. (A) MRI T2-weighted image with fat suppression performed 35 days after the onset of pancreatitis revealed a walled-off necrosis (arrow) in the pancreatic body, as well as residual inflammatory changes around the pancreatic tail (arrowheads). (B) Post-contrast arterial phase image showing a 1.8-cm pseudoaneurysm (arrow) arising from the splenic artery. Note the walled-off necrosis containing a mural thrombus is not enhanced (arrowhead), and peripancreatic walled-off necrosis (asterisks) can also be seen. (C) Pre-embolization selective arterial angiography showing pseudoaneurysm (arrow) arising from the pancreatic dorsal artery (a branch of the middle part of the splenic artery). (D) Post-embolization angiography showing disappearance of the lesion due to the resultant polyvinyl acetate particle embolization. MRI, magnetic resonance imaging.

embolization, might be applied.

Based on CT studies, portosystemic thrombosis has always been considered as a major late-phase vascular complication of AP (22-24). However, in our study, MRI detected splenic vein thrombosis in $44.4 \%$ of patients in the early phase of AP and in $55.6 \%$ patients in the late phase. The mean time for the detection of portosystemic thrombosis after diagnosis in AP patients differs across imaging modalities. Recently, Ding et al. (25) found the mean time for portosplenomesenteric thrombosis to be detected by CT was about 6 days; however, because their study only enrolled patients in the early phase of pancreatitis, this duration may have been shortened artificially. Our results are similar to those of Dörffel et al. (26), who reported that portal venous thromboses were demonstrated by ultrasonography at an average time of 10-14 days (range, 3 days to 7 weeks) after diagnosis. We also observed that patients with splenic vein thrombosis determined in the late phase of AP showed signs of splenic vein phlebitis on their initial MRI scan. This new finding was probably due to early-phase vein inflammation, which eventually damages the endothelium, rendering the vessel susceptible to late-phase thrombosis $(20,27)$. Also, patients with splenic vein thrombosis detected by initial MRI exhibited thrombus absorption on repeat MRI after treatment. These findings suggest that MRI could be a favorable technique for the evaluation of vascular condition improvement during the treatment period.

Another strength of our study is that we evaluated the relationship between the prevalence of various types of involvement and the severity of AP, as determined by the MRSI, with a correlation between the two having been reported previously (20). The strongest correlation with severity was seen in splenic vein phlebitis $(\mathrm{r}=0.532)$, 
A

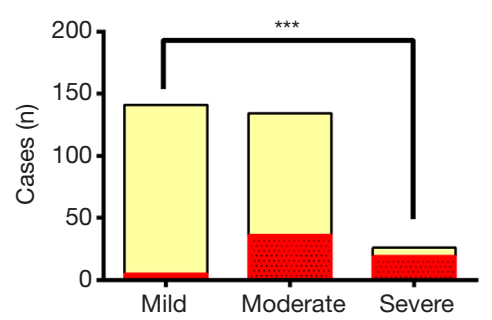

Desenterica superior vein phlebitis

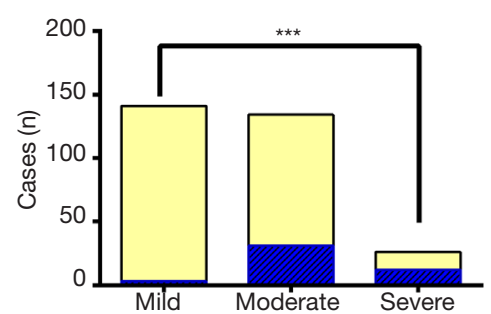

G

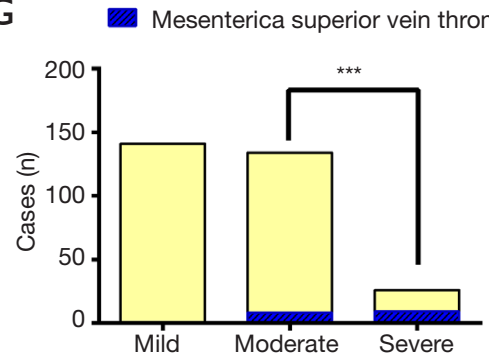

B

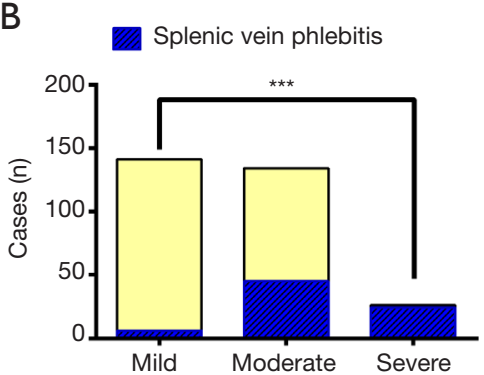

E

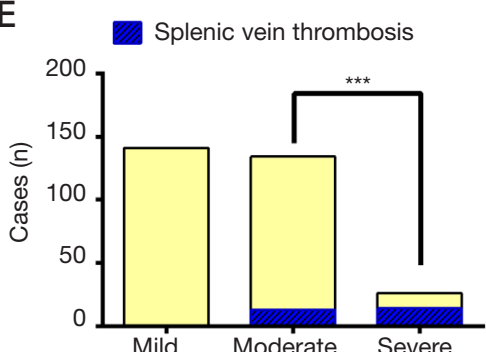

H Portal hypertension/varices

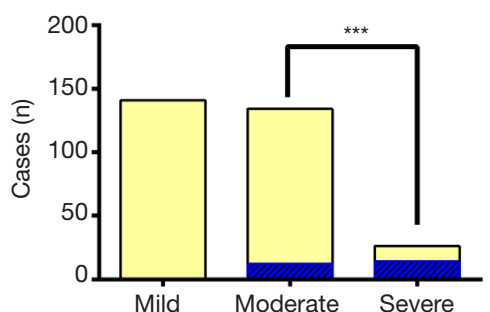

C

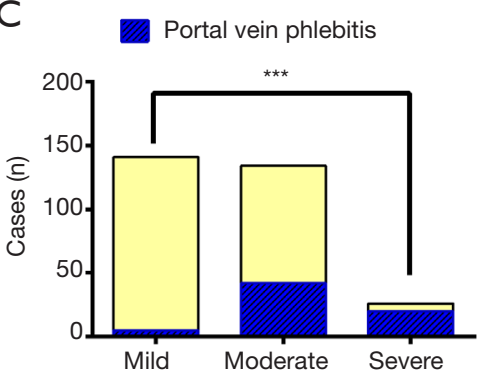

$\mathrm{F}$

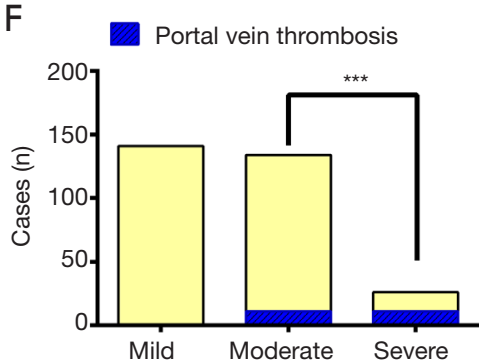

I

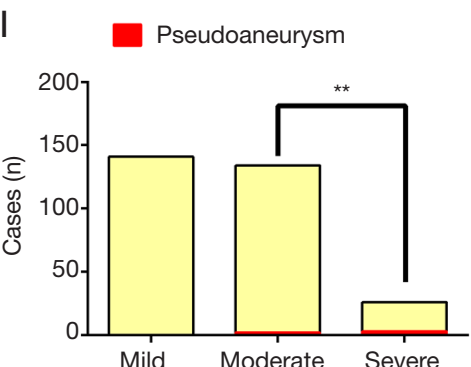

Figure 6 Comparison of the prevalence of each type of vascular involvement in mild, moderate, and severe acute pancreatitis. ${ }^{* *}, \mathrm{P}<0.01$; ***, $\mathrm{P}<0.001$.

Table 3 Correlation of the prevalence of each vascular complication in acute pancreatitis with the severity determined by the MRSI

\begin{tabular}{|c|c|c|c|c|}
\hline Peripancreatic vascular involvement & \multicolumn{3}{|c|}{ AP type (based on MRSI) } & $\mathrm{R}$ \\
\hline Splenic arterial arteritis, \% [n] & $3.5[5]$ & $26.9[36]$ & $73.1[19]$ & $0.456^{\#}$ \\
\hline Splenic vein phlebitis, \% [n] & $4.3[6]$ & $33.6[45]$ & $92.3[24]$ & $0.532^{\#}$ \\
\hline Portal vein phlebitis, \% [n] & $3.5[5]$ & $31.3[42]$ & 76.9 [20] & $0.487^{\#}$ \\
\hline Splenic vein thrombosis, \% [n] & 0 & $9.7[13]$ & $53.8[14]$ & $0.435^{\#}$ \\
\hline Portal vein thrombosis, $\%[n]$ & 0 & $8.2[11]$ & $42.3[11]$ & $0.365^{\#}$ \\
\hline Mesenteric superior venous thrombosis, \% [n] & 0 & $6.0[8]$ & $34.6[9]$ & $0.343^{\#}$ \\
\hline Portal hypertension/varices, \% [n] & 0 & $9.7[13]$ & $53.8[14]$ & $0.435^{\#}$ \\
\hline
\end{tabular}

During the hospital stay, two patients died due to permanent organ dysfunction. All other patients recovered well after treatment. ", $\mathrm{P}<0.001 ;{ }^{\&}, \mathrm{P}=0.007$. AP, acute pancreatitis; MRSI, MR severity index. 
followed by portal vein phlebitis $(\mathrm{r}=0.487)$, and splenic arterial involvement/arteritis $(\mathrm{r}=0.456)$. These results suggested that MRI detection of early vascular involvement can better predict the severity of AP as compared to other imaging approaches, which is in agreement with the findings of other studies $(20,25)$. Furthermore, similar findings using the clinical APACHE II system probably explain that vascular situations in AP also help evaluate the severity of pancreatitis (28).

One limitation of the current study is that most MRIdetectable vascular abnormalities in AP do not carry a gold standard of angiography, because the latter approach is invasive and involves a potential risk for AP patients. Compared with angiography, MRI is less sensitive for assessing vessel injury; however, as highlighted in this study, MRI has the ability to detect vessel damage. Another limitation is the retrospective nature of the study, which means there may be a potential sample selection bias. Usually, patients with moderately severe or severe AP follow a clinical course with two phases, and this may involve repeat MRI in the late phase. However, mild AP patients can also be hospitalized for longer than 7 days, and they too might need to undergo repeat MRI for treatment evaluation in our institution. Based on our results, mild, moderate, and severe pancreatitis, as determined by the MRSI, was seen in 46.8\% (141/301), 44.6\% (134/301), and $8.6 \%(26 / 301)$ of patients in our study, respectively. Therefore, the potential sample selection of mild and more severe patients was relatively balanced. In our study, the 125 patients with necrotizing pancreatitis included 120 patients with mixed pancreatic necrosis and only 5 patients with extrapancreatic necrosis alone. As no patients in our cohort had pancreatic parenchymal necrosis alone, we did not analyze the differences in vascular involvement among the three subtypes of necrosis in this study. Future studies should investigate the differences between the three subtypes, and a multivariate analysis should be performed on the severity of pancreatitis according to the type of peripancreatic vascular involvement in a larger prospective cohort.

In conclusion, our results show that vascular abnormalities associated with AP are relatively common, particularly in the early phase of the disease. We described the main MRI features of these abnormalities and emphasized the importance of recognizing early signs, such as splenic vein phlebitis and arterial involvement/arteritis. The findings of this study highlight the utility of MRI in revealing the extent of the inflammatory process and subsequent vascular progression on repeat MRI during treatment. More importantly, vascular involvement detected by MRI in the early stage may be useful for reflecting the severity of AP in the clinical setting.

\section{Acknowledgments}

Funding: This work was funded by the Nanchong City College Cooperative Research Project (No. 19SXHZ0282); Sichuan Medical Youth Innovation Research Project (No. Q19067).

\section{Footnote}

Conflicts of Interest: All authors have completed the ICMJE uniform disclosure form (available at http://dx.doi. org/10.21037/qims-20-280). The authors have no conflicts of interest to declare.

Ethical Statement: This study was conducted in accordance with the Declaration of Helsinki (as revised in 2013) and was approved by institutional ethics board of Affiliated Hospital of North Sichuan Medical College (NO.: 2017ER(A)026). Informed consent was waived, as the study was retrospective in design.

Open Access Statement: This is an Open Access article distributed in accordance with the Creative Commons Attribution-NonCommercial-NoDerivs 4.0 International License (CC BY-NC-ND 4.0), which permits the noncommercial replication and distribution of the article with the strict proviso that no changes or edits are made and the original work is properly cited (including links to both the formal publication through the relevant DOI and the license). See: https://creativecommons.org/licenses/by-nc-nd/4.0/.

\section{References}

1. Banks PA, Bollen TL, Dervenis C, Gooszen HG, Johnson CD, Sarr MG, Tsiotos GG, Vege SS; Acute Pancreatitis Classification Working Group. Classification of acute pancreatitis 2012: revision of the Atlanta classification and definitions by international consensus. Gut 2013;62:102-11.

2. Thoeni RF. The revised Atlanta classification of acute pancreatitis: its importance for the radiologist and its effect on treatment. Radiology 2012;262:751-64.

3. Sheu Y, Furlan A, Almusa O, Papachristou G, Bae KT. The revised Atlanta classification for acute pancreatitis: a CT imaging guide for radiologists. Emerg Radiol 2012;19:237-43.

4. Bollen TL, Singh VK, Maurer R, Repas K, van Es HW, Banks PA, Mortele KJ. A comparative evaluation of radiologic and clinical scoring systems in the early 
prediction of severity of acute pancreatitis. Am J

Gastroenterol 2012;107:612-9.

5. Xiao B, Xu HB, Jiang ZQ, Zhang J, Zhang XM. Current concepts for the diagnosis of acute pancreatitis by multiparametric magnetic resonance imaging. Quant Imaging Med Surg 2019;9:1973-85.

6. Shyu JY, Sainani NI, Sahni VA, Chick JF, Chauhan NR, Conwell DL, Clancy TE, Banks PA, Silverman SG. Necrotizing pancreatitis: diagnosis, imaging, and intervention. Radiographics 2014;34:1218-39.

7. Mortelé KJ, Mergo PJ, Taylor HM, Wiesner W, Cantisani V, Ernst MD, Kalantari BN, Ros PR. Peripancreatic vascular abnormalities complicating acute pancreatitis: contrastenhanced helical CT findings. Eur J Radiol 2004;52:67-72.

8. Tang MY, Chen TW, Bollen TL, Wang YX, Xue HD, Jin ZY, Huang XH, Xiao B, Li XH, Ji YF, Zhang XM. MR imaging of hemorrhage associated with acute pancreatitis. Pancreatology 2018;18:363-9.

9. Türkvatan A, Erden A, Türkoğlu MA, Seçil M, Yüce G. Imaging of acute pancreatitis and its complications. Part 2: complications of acute pancreatitis. Diagn Interv Imaging 2015;96:161-9.

10. Xiao B, Zhang XM, Tang W, Zeng NL, Zhai ZH. Magnetic resonance imaging for local complications of acute pancreatitis: a pictorial review. World J Gastroenterol 2010;16:2735-42.

11. Balthazar EJ. Complications of acute pancreatitis: clinical and CT evaluation. Radiol Clin North Am 2002, 40:1211-27.

12. Meyrignac O, Lagarde S, Bournet B, Mokrane FZ, Buscail L, Rousseau H, Otal P. Acute Pancreatitis: Extrapancreatic Necrosis Volume as Early Predictor of Severity. Radiology 2015;276:119-28.

13. Sun H, Zuo HD, Lin Q, Yang DD, Zhou T, Tang MY, Wáng YX, Zhang XM. MR imaging for acute pancreatitis: the current status of clinical applications. Ann Transl Med 2019;7:269.

14. Yang R, Jing ZL, Zhang XM, Tang W, Xiao B, Huang XH, Yang L, Feng ZS. MR imaging of acute pancreatitis: correlation of abdominal wall edema with severity scores. Eur J Radiol 2012;81:3041-7.

15. Tenconi SM, Rausei S, Boni L, Dionigi G, Rovera F. Preoperative splenic vein diameter: a risk factor for portalsplenic vein thrombosis after laparoscopic splenectomy? Surgery 2010;148:164.

16. Xie CL, Wu CQ, Chen Y, Chen TW, Xue HD, Jin ZY, Zhang XM. Sinistral Portal Hypertension in Acute Pancreatitis: A Magnetic Resonance Imaging Study. Pancreas 2019;48:187-92.

17. Ferraz AA, Campos JM, de Araújo JG Jr, de Carvalho MR, Neto JP, Ferraz EM. Influence of the inversion of the portal/splenic vein diameter in the results of the surgical treatment of schistossomotic portal hypertension. Rev Col Bras Cir 2011;38:35-40.

18. Yadav D, Lowenfels AB. The epidemiology of pancreatitis and pancreatic cancer. Gastroenterology 2013;144:1252-61.

19. Lankisch PG, Apte M, Banks PA. Acute pancreatitis. Lancet 2015;386:85-96.

20. Xie CL, Zhang M, Chen Y, Hu R, Tang MY, Chen TW, Xue HD, Jin ZY, Zhang XM. Spleen and splenic vascular involvement in acute pancreatitis: an MRI study. Quant Imaging Med Surg 2018;8:291-300.

21. Bergert H, Hinterseher I, Kersting S, Leonhardt J, Bloomenthal A, Saeger HD. Management and outcome of hemorrhage due to arterial pseudoaneurysms in pancreatitis. Surgery 2005;137:323-8.

22. Harris S, Nadkarni NA, Naina HV, Vege SS. Splanchnic vein thrombosis in acute pancreatitis: a single-center experience. Pancreas 2013;42:1251-4.

23. Toqué L, Hamy A, Hamel JF, Cesbron E, Hulo P, Robert S, Aube C, Lermite E, Venara A. Predictive factors of splanchnic vein thrombosis in acute pancreatitis: A 6-year single-center experience. J Dig Dis 2015;16:734-40.

24. Jiang W, Zhou J, Ke L, Li G, Tong ZH, Li WQ, Li JS. Splanchnic vein thrombosis in necrotizing acute pancreatitis: Detection by computed tomographic venography. World J Gastroenterol 2014;20:16698-701.

25. Ding L, Deng F, Yu C, He WH, Xia L, Zhou M, Huang X, Lei YP, Zhou XJ, Zhu Y, Lu NH. Portosplenomesenteric vein thrombosis in patients with early-stage severe acute pancreatitis. World J Gastroenterol 2018;24:4054-60.

26. Dörffel T, Wruck T, Rückert RI, Romaniuk P, Dörffel Q, Wermke W. Vascular complications in acute pancreatitis assessed by color duplex ultrasonography. Pancreas 2000;21:126-33.

27. Balthazar EJ, Fisher LA. Hemorrhagic complications of pancreatitis: radiologic evaluation with emphasis on CT imaging. Pancreatology 2001;1:306-13.

28. Inoue K, Hirota M, Beppu T, Ishiko T, Kimura Y, Maeda K, Ogawa M. Angiographic features in acute pancreatitis: the severity of abdominal vessel ischemic change reflects the severity of acute pancreatitis. JOP 2003;4:207-13.

Cite this article as: Jiang ZQ, Xiao B, Zhang XM, Xu HB. Early-phase vascular involvement is associated with acute pancreatitis severity: a magnetic resonance imaging study. Quant Imaging Med Surg 2021;11(5):1909-1920. doi: 10.21037/ qims-20-280 